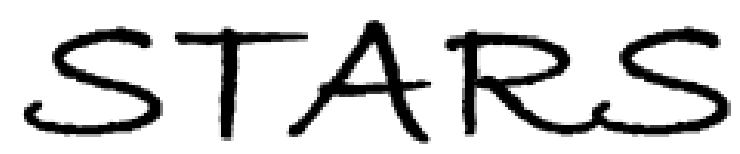

University of Central Florida

STARS

Faculty Bibliography 1990s

Faculty Bibliography

$1-1-1999$

\title{
Actively mode-locked p-Ge laser in Faraday configuration
}

\author{
A. V. Muravjov \\ University of Central Florida \\ S. H. Withers \\ University of Central Florida \\ R. C. Strijbos \\ University of Central Florida \\ S. G. Pavlov \\ V. N. Shastin
}

See next page for additional authors

Find similar works at: https://stars.library.ucf.edu/facultybib1990

University of Central Florida Libraries http://library.ucf.edu

This Article is brought to you for free and open access by the Faculty Bibliography at STARS. It has been accepted for inclusion in Faculty Bibliography 1990s by an authorized administrator of STARS. For more information, please contact STARS@ucf.edu.

\section{Recommended Citation}

Muravjov, A. V.; Withers, S. H.; Strijbos, R. C.; Pavlov, S. G.; Shastin, V. N.; and Peale, R. E., "Actively modelocked p-Ge laser in Faraday configuration" (1999). Faculty Bibliography 1990s. 2758.

https://stars.library.ucf.edu/facultybib1990/2758

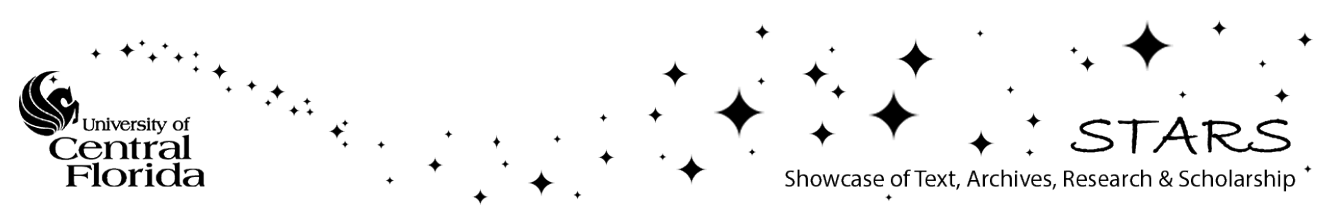




\section{Authors}

A. V. Muravjov, S. H. Withers, R. C. Strijbos, S. G. Pavlov, V. N. Shastin, and R. E. Peale 


\section{Actively mode-locked $\mathrm{p}-\mathrm{Ge}$ laser in Faraday configuration}

Cite as: Appl. Phys. Lett. 75, 2882 (1999); https://doi.org/10.1063/1.125179

Submitted: 17 May 1999. Accepted: 13 September 1999. Published Online: 03 November 1999

A. V. Muravjov, S. H. Withers, R. C. Strijbos, S. G. Pavlov, V. N. Shastin, and R. E. Peale
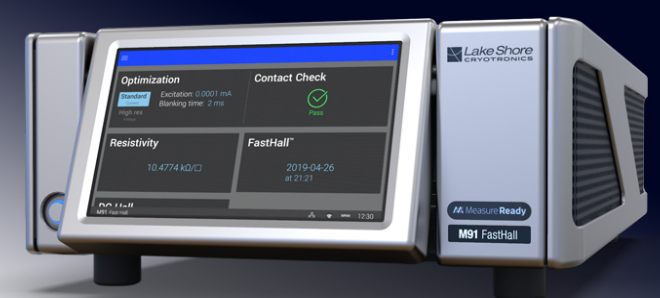

\section{Measure Ready} M91 FastHall ${ }^{\text {TM }}$ Controller

A revolutionary new instrument for complete Hall analysis

\section{See the video 0}




\title{
Actively mode-locked $\boldsymbol{p}$-Ge laser in Faraday configuration
}

\author{
A. V. Muravjov, S. H. Withers, and R. C. Strijbos \\ Department of Physics, University of Central Florida, Orlando, Florida 32816 \\ S. G. Pavlov and V. N. Shastin \\ Institute for the Physics of Microstructures, Russian Academy of Sciences, Nizhny, Novgorod, Russia \\ R. E. Peale $e^{a)}$ \\ Department of Physics, University of Central Florida, Orlando, Florida 32816
}

(Received 17 May 1999; accepted for publication 13 September 1999)

\begin{abstract}
Active mode locking of the far-infrared $p$-Ge laser has been achieved in the Faraday configuration of electric and magnetic fields applied to the laser crystal. The laser generates 200 ps pulses of $80-110 \mathrm{~cm}^{-1}$ radiation with a laser-cavity roundtrip frequency of $454 \mathrm{MHz}$. The mechanism of gain modulation by the external rf electric field is based on induced electric-field gradients inside the active crystal and requires less rf power than was found previously for Voigt geometry. (C) 1999 American Institute of Physics. [S0003-6951(99)01045-1]
\end{abstract}

Far-infrared $p$-Ge lasers operate in the wavelength range from 70 to $200 \mu \mathrm{m} .{ }^{1}$ The usual pulse duration is a few $\mu \mathrm{s}$ with usual peak powers of 1-10 W. Stimulated emission occurs on direct optical transitions between light- and heavyhole valence subbands in bulk $p$-Ge at liquid helium temperatures in strong crossed electric $\mathbf{E}$ and magnetic $\mathbf{B}$ fields. Population inversion is built up via light hole accumulation at the optimal ratio $E / B$, when heavy holes repeatedly emit an optical phonon after being accelerated beyond the threshold energy $37 \mathrm{meV}$, but light holes move on closed cyclotron orbits below this threshold and have a much longer lifetime. A large gain bandwidth $(\Delta \omega / \omega \sim 1)$ and low dispersion make this active medium promising for wide range tunability and for amplification and generation of short pulses of farinfrared radiation with picosecond duration $\tau \sim 1 / \Delta \omega$.

In previous papers, ${ }^{2-5}$ generation of $200 \mathrm{ps}$ far-infrared pulses by the $p$-Ge laser was obtained by active mode locking in Voigt geometry of applied fields with gain modulation at one end of the laser crystal. The modulation field $E_{\mathrm{rf}}$ was applied at a frequency $\nu_{\text {rf }}$ equal to the half of the cavity roundtrip frequency $\nu_{\mathrm{rf}}$. According to the proposed mechanism of gain modulation, ${ }^{6} E_{\mathrm{rf}}$ applied parallel to the magnetic field periodically accelerates light holes beyond the optical phonon threshold, where they are predominantly scattered to the heavy-hole subband. As a consequence, the gain is modulated at the roundtrip frequency, inducing mode locking. This mechanism requires Voigt geometry of applied $\mathbf{E} \perp \mathbf{B}$ fields, where $\mathbf{E}$ and $\mathbf{B}$ are both perpendicular to the optical axis of the active crystal (direction of light propagation). The modulating field $\mathbf{E}_{\mathrm{rf}} \| \mathbf{B}$ can be applied at one end of the active crystal directly through small ohmic contacts on the lateral sides. In this letter we demonstrate active mode locking of a $p$-Ge laser in Faraday geometry, where the magnetic field is applied along the long optical axis of the sample, and the orientation of the applied modulating field $\mathbf{E}_{\mathrm{rf}}$ is perpendicular to $\mathbf{B}$ (Fig. 1).

Single-crystal, Ga-doped $p$-Ge with a concentration of

${ }^{a)}$ Electronic mail: rep@physics.ucf.edu
$7 \times 10^{13} \mathrm{~cm}^{-3}$ was cut into the form of a rectangular bar with a cross section of $5 \times 7 \mathrm{~mm}^{2}$ and a length of $84.2 \mathrm{~mm}$. The crystal ends were polished parallel to each other within 1 arcmin accuracy and two external copper mirrors were attached to them via $20 \mu \mathrm{m}$ Teflon film. Electric field pulses $\mathbf{E}$ were applied to the active crystal using a low duty-cycle thyratron pulser via ohmic contacts on opposite 5 $\times 84.2 \mathrm{~mm}^{2}$ sides of the crystal. Contacts were made by $\mathrm{Al}$ evaporation, subsequent annealing, and coating with In. The active crystal with attached mirrors was installed inside a superconducting solenoid with tunable magnetic field up to 3 $\mathrm{T}$ oriented along the long crystal axis, such that the applied field orientations were $\mathbf{E} \|[1-10]$ and $\mathbf{B} \|[111]$. The entire system is immersed in liquid helium at $4 \mathrm{~K}$. Radiation was conducted out of the top of the cryostat using a brass light pipe sealed with a Teflon lens. The laser emission was detected by a fast Schottky diode (University of Virginia) and recorded on a $4.5 \mathrm{GHz}$ transient digitizer (Tektronix SCD5000).

A few pairs of small additional contacts with a length of $4 \mathrm{~mm}$ and $1 \mathrm{~mm}$ separation where evaporated on the lateral sides of the crystal (Fig. 1). In these experiments, the exter-

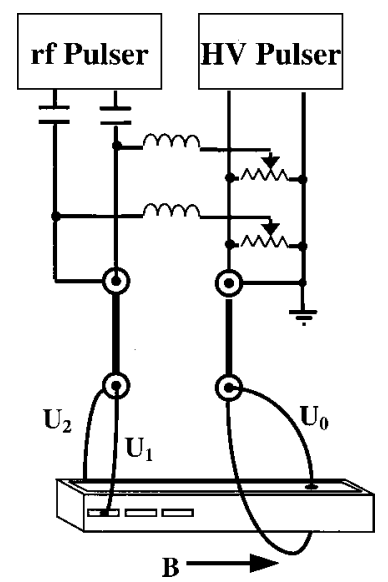

FIG. 1. Contact configuration with excitation and modulation electronics for a mode-locked $p$-Ge laser. Thick lines denote coaxial cables. 


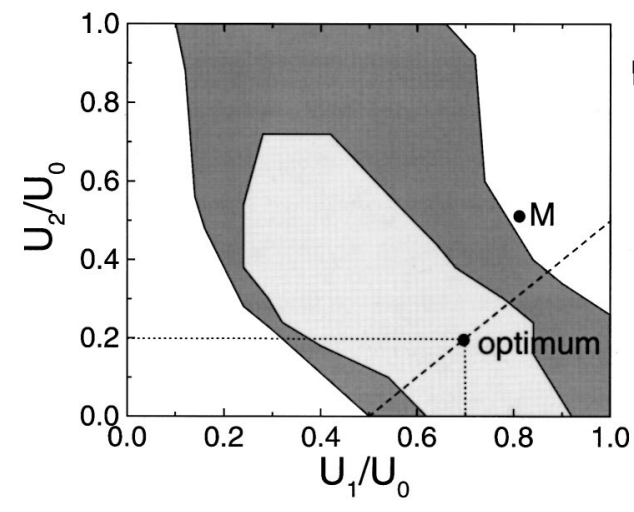

FIG. 2. Intensity contours vs normalized bias potentials at the additional contacts. The diagonal dashed line indicates the condition $U_{1}-U_{2}=U_{\text {Hall }}$. The optimum point indicates the bias values for best lasing. Point $\mathrm{M}$ indicates the bias values for the best mode locking effect.

nal $454 \mathrm{MHz}$ rf electric field was applied to the contact pair nearest to the back mirror so that the applied $\mathbf{E}_{\mathrm{rf}}$ is perpendicular to both $\mathbf{E}$ and $\mathbf{B}$. The electronic setup (Fig. 1) allows control of the bias potentials on the additional contacts. The rf circuit is described in Ref. 5.

Figure 2 shows the intensity contours of the laser operation as a function of the bias voltages $U_{1}$ and $U_{2}$ on the additional contacts. The laser does not operate outside the outer contour in the unshaded region. Without applying external bias, $U_{1}$ and $U_{2}$ have values determined by the Hall effect, which corresponds to the "optimum" point for the gain.

The mechanism of active mode locking is local modulation of the gain inside the active crystal by applying a rf electric field $\mathbf{E}_{\mathrm{rf}}$. The best active mode locking effect was found at the bias conditions indicated by the point labeled $\mathrm{M}$ in Fig. 2. The laser does not operate here without the additional rf field $\mathbf{E}_{\mathrm{rf}}$ and starts to operate only when $\mathbf{E}_{\mathrm{rf}}$ is applied exactly at the laser-cavity roundtrip frequency 454 $\pm 0.5 \mathrm{MHz}$. The laser emission consists of a train of $200 \mathrm{ps}$ mode-locked pulses, as shown in Fig. 3. The $1 \mathrm{MHz}$ width of the resonance is defined by the build-up time for stimulated emission of a few hundred nanoseconds.

In our opinion, the local gain modulation is induced by the appearance of a longitudinal $E$-field component along B in the center of the sample when external voltage is applied to the additional contacts, as shown schematically in Fig. 4.

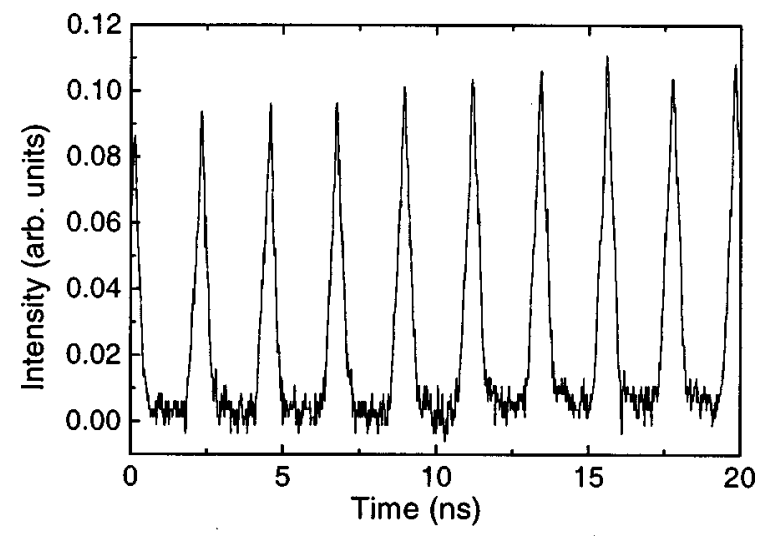

FIG. 3. Mode-locked output of a Faraday-configured $p$-Ge laser. The peak widths are bandwidth limited by the transient digitizer.

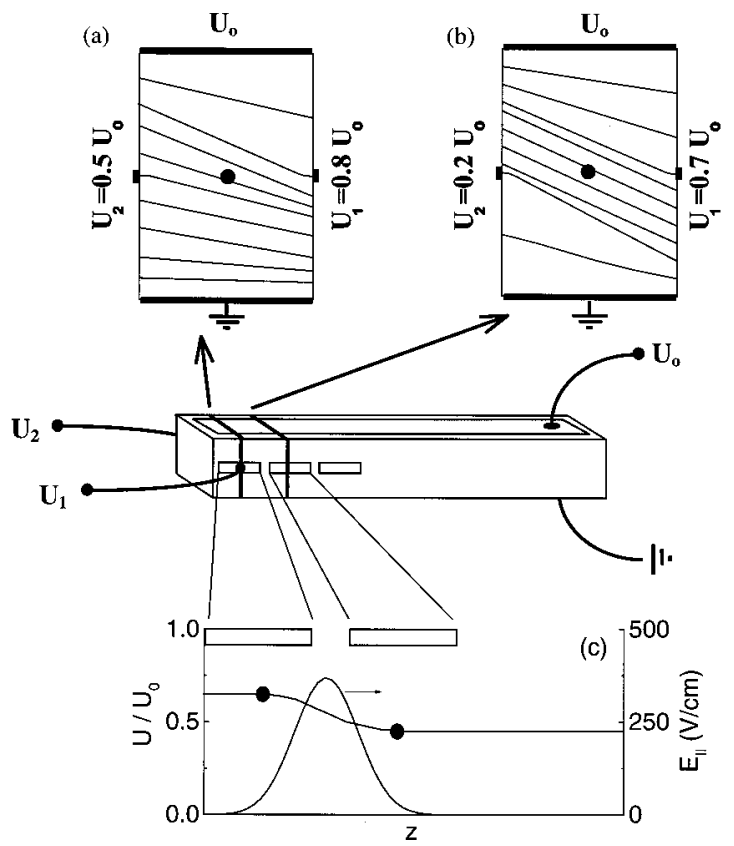

FIG. 4. Schematic of electric potential distributions inside the $p$-Ge laser crystal. (a) Cross sectional slice under the first pair of additional contacts with equipotential contours in units of $0.1 U_{0}$. The $U_{1}$ and $U_{2}$ bias values correspond to point $\mathrm{M}$ in Fig. 2. (b) Cross sectional slice under the second pair of additional contacts without external bias. $U_{1}$ and $U_{2}$ assume values close to the optimum point in Fig. 2. (c) Potential vs distance along the main crystal axis and the corresponding electric field $E_{\|}$.

Potentials $U_{1}$ and $U_{2}$ corresponding to point M (Fig. 2) are applied to the first pair of additional contacts [Fig. 4(a)], and the potentials on the second pair of contacts are controlled only by the Hall field. Dot symbols represent the potential values at the center of the sample cross section. The potential gradient along $z$ [Fig. 4(c)] corresponds to the appearance of a local electric field $E_{\|}$in the direction of the magnetic field, which decreases the local gain. When a rf electric field is applied to the additional contacts, the potential at the center of the crystal cross section is modulated at the rf frequency, modulating the potential gradient along $B$, and finally the local gain.

The possibility of short pulse generation by active mode locking of the $p$-Ge laser in Faraday geometry has not been discussed prior to this letter, but this successful demonstration reveals a number of advantages. The magnetic field is conveniently applied by a compact, low-current superconducting solenoid with easily controlled field magnitude. The train of 200 ps output pulses was found to be more repeatable and stable than in the conventional Voigt geometry.

Another advantage of Faraday geometry for gain modulation is the relatively high impedance and thus low current between additional contacts. In Voigt configuration, the impedance between rf contacts was estimated to be only $\sim 1-5$ $\Omega .^{2-5}$ In Faraday geometry, the contribution of magnetoresistance increases this value to $\sim 50 \Omega$, which is the ideal load for most commercial electronics. Hence the required rf modulating-field power is lower in Faraday than in Voigt geometry. The measured level of reflected rf power was less than $20 \%$ without special impedance matching tricks. The suggested gain modulation mechanism can be optimized to further reduce the required rf power and to generate shorter pulses. 
In conclusion, active mode locking of a $p$-Ge laser in Faraday configuration of applied crossed electric and magnetic fields has been demonstrated. The postulated mechanism of local gain modulation is based on oscillation of induced local potential gradients along the magnetic field. This scheme of active mode locking is applicable for all traditional $p$-Ge lasers with Faraday configuration of applied $\mathbf{E} \perp \mathbf{B}$ fields.

This work was supported by the NSF and BMDO. The authors from the Institute for the Physics of Microstructures thank the Russian Foundation for Basic Research.
${ }^{1}$ Special Issue on Far-infrared Semiconductor Lasers, edited by E. Gornik and A. A. Andronov, Opt. Quantum Electron. 23, (1991).

${ }^{2}$ J. N. Hovenier, A. V. Muravjov, S. G. Pavlov, V. N. Shastin, R. C. Strijbos, and W. Th. Wenkebach, Appl. Phys. Lett. 71, 443 (1997).

${ }^{3}$ J. N. Hovenier, T. O. Klaassen, W. Th. Wenckebach, A. V. Muravjov, S. G. Pavlov, and V. N. Shastin, Appl. Phys. Lett. 72, 1140 (1998).

${ }^{4}$ A. V. Muravjov, R. C. Strijbos, C. J. Fredricksen, H. Weidner, W. Trimble, A. Jamison, S. G. Pavlov, V. N. Shastin, and R. E. Peale, in Radiative Processes and Dephasing in Semiconductors, OSA-TOPS, Vol. 18, edited by D. Citron (OSA, Washington, DC, 1998), pp. 102-107.

${ }^{5}$ A. V. Muravjov, R. C. Strijbos, C. J. Fredricksen, S. H. Withers, W. Trimble, S. G. Pavlov, V. N. Shastin, and R. E. Peale, Appl. Phys. Lett. 74, 167 (1999).

${ }^{6}$ R. C. Strijbos, J. G. S. Lok, and W. Th. Wenckebach, J. Phys.: Condens. Matter 6, 7461 (1994). 